\title{
Contrastive Analysis of Car Interior Sound Quality Evaluation Models
}

\author{
Yanyan Zuo ${ }^{1}$,Lianying Liao ${ }^{1,2,} \mathrm{a}^{*}$, Haodong Meng ${ }^{2}$ and Saisai $\mathrm{Wu}^{1}$ \\ ${ }^{1}$ Institute of Noise and Vibration, Jiangsu University, Zhenjiang, 212013, China \\ ${ }^{2}$ Changzhou Institute of Technology, Changzhou, 213002, China \\ a liaolianying@163.com
}

Keywords: car interior sound quality; objective evaluation model; MLR (Multiple Linear Regression); BP neural network; radial basis function

\begin{abstract}
Sound quality evaluation may be conducted with multiple different objective evaluation models, but reliability of evaluation results is different. Noise samples of two ears of the driver were gathered when the car operated at the speed of $60 \mathrm{~km} / \mathrm{h}$ and different music was played in the car. After equal loudness, frequency band and filtering treatment of sound samples, subjective evaluation of sound quality was carried out. Based on calculation of objective parameters, MLR (Multiple Linear Regression) objective evaluation model for sound quality, objective evaluation model for sound quality based on BP neural network and objective evaluation model for sound quality based on RBF (radial basis function) neural network were established. The test samples were substitute into the three models, and their sound quality prediction effects at each frequency band were compared. The results show that MLR model is simple and has fast calculation speed, so it is suitable for linear problem analysis; BP neural network model is suitable for solving large sample problem with complex internal mechanism; RBF neural network model has high precision and small relative error, so it is suitable for analysis of nonlinear problem of small sample. Under experimental conditions, prediction effect of RBF model is superior to MLR model and BP neural network model.
\end{abstract}

\section{Introduction}

As the researches on noise gradually go deep, it is found that A-weighted sound pressure level (SPL) of some noises is very low, but human subjective feeling is not comfortable [1]. This indicates human subjective feeling for sound is also influenced by other sound parameters. Sound quality can well reflect human subjective feeling for noises in specific environment. Sound quality accurately considers human psychological response mechanism and noise perception characteristic and can reflect subjective feeling differences caused by different noise signals [2].

The precondition of sound quality research is to establish standard evaluation system, including subjective evaluation and objective evaluation. Sound cannot be seen and touched and involves human physiological and psychological response which belongs to subjective feeling. In practical application, almost all sound evaluations are based on subjective evaluation and assisted by objective evaluation. So far, no instrument can accurately simulate the response of human ear to sound. No analysis method can be used to simulate human subjective feeling. Due to the two reasons, the situation where sound quality is judged by human ear will continue for a quite long time. But subjective evaluation experiment needs to consume more human power and time, so objective evaluation is needed for auxiliary research. In recent years, researchers have proposed various objective quantitative models to describe human subjective evaluation. Objective parameters are based on psychological acoustic parameters. But most often, the correlation between 
some psychological acoustic parameters and subjective evaluation results of sound quality is not high. The major reason is that different objective quantitative models have different application conditions. It thus can be seen that to gain reliable sound quality evaluation effect, how to choose correct objective quantitative models is particularly important. MLR, BP neural network and radial basis function are frequently applied to establish objective evaluation models for sound quality. This paper combines examples to compare prediction results of the three models at each frequency band and summarizes advantages, disadvantages and application conditions of the three evaluation models in sound quality evaluation.

\section{Sound sample collection and pretreatment}

Sound signal collection. The experiment was conducted by referring to GB/T18697-2002. An open site was chosen and more than $20 \mathrm{~m}$ away from buildings and other large objects. Except the driver, testing personnel and testing equipment, the car had no other load. All car windows were closed, and auxiliary device stopped working. In the experiment, the driver wore a set of microphone, and the co-driver held HEAD portable sound analysis instrument Squadriga I in hand. The sampling frequency was $2.4 \mathrm{KHz}$. The sound signals were gathered when the car ran at the speed of $60 \mathrm{~km} / \mathrm{h}$ and 26 different styles of music were played in the car. 2 seriously-disturbed sound signals were eliminated through subjective comparison, and 24 sound sample songs including low-frequency noise in the car were gained [3,4].

Sound sample pretreatment. Sound samples gathered concentrate between $40 \mathrm{~dB}$ and $60 \mathrm{~dB}$. Different loudness exerts a tremendous influence on human subjective feeling [5]. Equal-loudness treatment was conducted for 24 sound samples by referring to the sound samples with loudness value of 20sone. Table 1 lists changes of each parameter of sound sample 2 and 6 after and before equal-loudness treatment. Prior to correction, N2\#<20sone and N6\#>20sone. After correction, N2\# and N6\# are about 20sone. Thus, subjective evaluation error caused by loudness can be effectively avoided. It is found through comparison that, sharpness, harshness, fluctuation and tone basically have positive correlation with loudness. In essence, equal-loudness amounts to a weighting function and will not change differences of each parameter while loudness is corrected [6].

Table 1 All the parameters of sample 2 and sample 6 before and after equal loudness process

\begin{tabular}{ccccc}
\hline Acoustic quantity & 2\# & 2\#amendment & 6\# & 6\# amendment \\
\hline Loudness & 17.8 & 19.7 & 25.95 & 20.15 \\
Sharpness & 1.08 & 1.115 & 2.035 & 1.895 \\
Roughness & 1.735 & 1.84 & 1.715 & 1.495 \\
Fluctuation & 0.047 & 0.049 & 0.059 & 0.053 \\
Tone & 0.079 & 0.078 & 0.266 & 0.274 \\
A-weighted SPL & 62.9 & 64.4 & 66.75 & 62.95 \\
\hline
\end{tabular}

Considering large loudness will lead to evaluators' fatigue and thus affect result accuracy, the sound samples were divided into four frequency bands to study the influence of frequency-division band on the whole sound quality: low and medium frequency $(20-160 \mathrm{~Hz})$, medium frequency $(160-1280 \mathrm{~Hz})$, medium and high frequency $(1280-4000 \mathrm{~Hz})$ and high frequency (above $4000 \mathrm{~Hz})$. Filtering was carried out for 24 samples at different frequency bands after equal-loudness treatment respectively, and 96 sample signals were gained at different frequency bands.

\section{Subjective evaluation of sound quality and objective parameter calculation}

Subjective evaluation of sound quality. For most psychological acoustics evaluation tests, 20 evaluators can gain relatively accurate results. In this paper, 24 ion-campus researchers and teachers with driving experience and familiarity with noise environment in the car were chosen as the subjects of evaluation. The proportion of male and female subjects is 3:1. Before subjective 
evaluation, audition training is required for the subjects of evaluation in order to unify subjective evaluation standards.

The evaluation score of subject of evaluation on each sound sample is added to gain preference evaluation value of each evaluator on the sound sample. SPSS software is applied to carry out correlation analysis of evaluation result of each subject of evaluation and evaluation result of other subjects. The evaluation results with correlation coefficient less than 0.7 are eliminated. Finally, evaluation results are normalized according to Formula (1).

$$
X=0.8 \frac{X_{\mathrm{i}}-X_{\min }}{X_{\text {max }}-X_{\text {min }}}+0.1
$$

$X \mathrm{i}$ is random evaluation score; $X \mathrm{~min}$ is the minimum in the same group; $X \max$ is the maximum in the same group. Scatter diagram and linear relation of the whole frequency band and frequency-division band are shown in Fig.1.
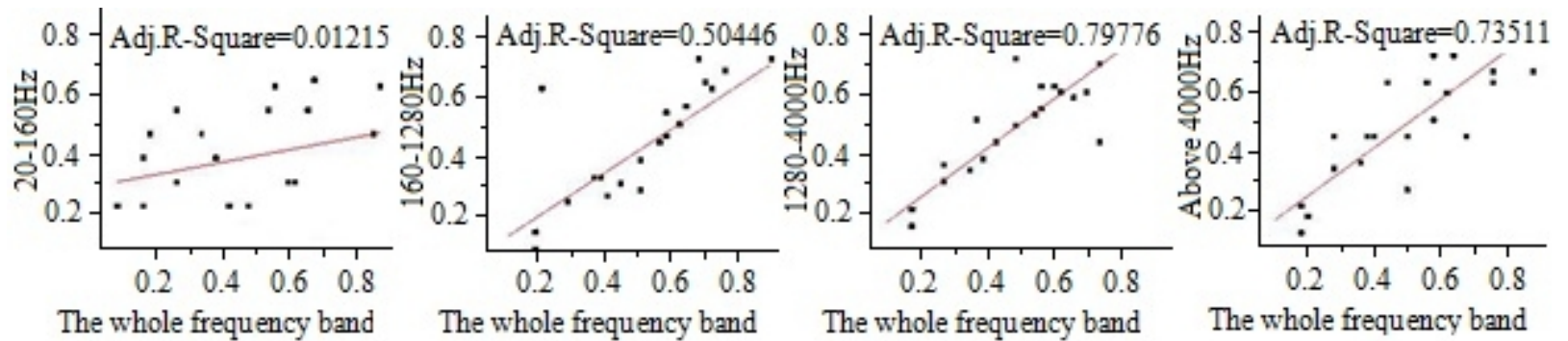

Fig.1. Scatter diagram and linear relationship

From Fig.1, it is known that correction determination coefficients Adj.R-Square of the whole frequency band with low-medium frequency, medium frequency, medium-high frequency and high frequency band are 0.01215, 0.50446, 0.79779 and 0.73511. The larger Adj.R-Square is, the lager determination coefficient $\mathrm{R}$. thus, subjective evaluation effect of $1280-4000 \mathrm{~Hz}$ frequency band on the whole frequency band is the largest. This frequency band mainly reflects tone pf samples. Secondly, high-frequency band above $4000 \mathrm{~Hz}$ has the closest relationship with speech articulation and speech intelligibility. But if the frequency band involves too many components, sharpness will be excessive so that people will generate auditory fatigue. Low-frequency samples mainly come from low-frequency noise in the car. Low-frequency component is very small, so auditory sense at $20-160 \mathrm{~Hz}$ is almost the same and the influence on the whole frequency band is also the smallest. In the following linear regression model, sound samples at $20-160 \mathrm{~Hz}$ are ignored.

Objective parameter calculation. Loudness Adaption software of HEAD Acoustics Company was applied to process noise samples of 24 pieces of music and they are saved as a new sound file. Then, ArtemiS 12.0 software was used to calculate objective parameter values of entire sound samples and signals in each frequency-division band. Objective parameters include 2 physical acoustical parameters (A-weighted sound pressure level and linear sound pressure level) and 6 psychological acoustical parameters (loudness, sharpness, harshness, fluctuation, AI index and tone) [7].

\section{Objective evaluation model of sound quality for interior car}

MLR-based sound quality evaluation model. Subjective evaluation values and objective parameter values of 1-18 sound samples are inputted in SPSS software. SPSS multivariate linear model for the whole frequency band, $160-1280 \mathrm{~Hz}, 1280-4000 \mathrm{~Hz}$ and above $4000 \mathrm{~Hz}$ is established. The model is larger and further close to 1 , which indicates fitting degree of regression equation is better [8]. Durbin-Watson statistical magnitude is about 2, which indicates the residual obeys normal distribution. If it deviates from 2 excessively, this means the model has no sufficient explanatory power. Fitting degree test of MLR model is shown in Table 2.

According to Table 2 Regression Coefficients (regression coefficients of only the whole frequency band are listed), MLR model of objective evaluation of noise samples in the car in the 
whole frequency band and each frequency band can be gained. It can be expressed with matrix, as shown in Formula (2).

Table 2 Fitting degree inspection of multiple linear regression model

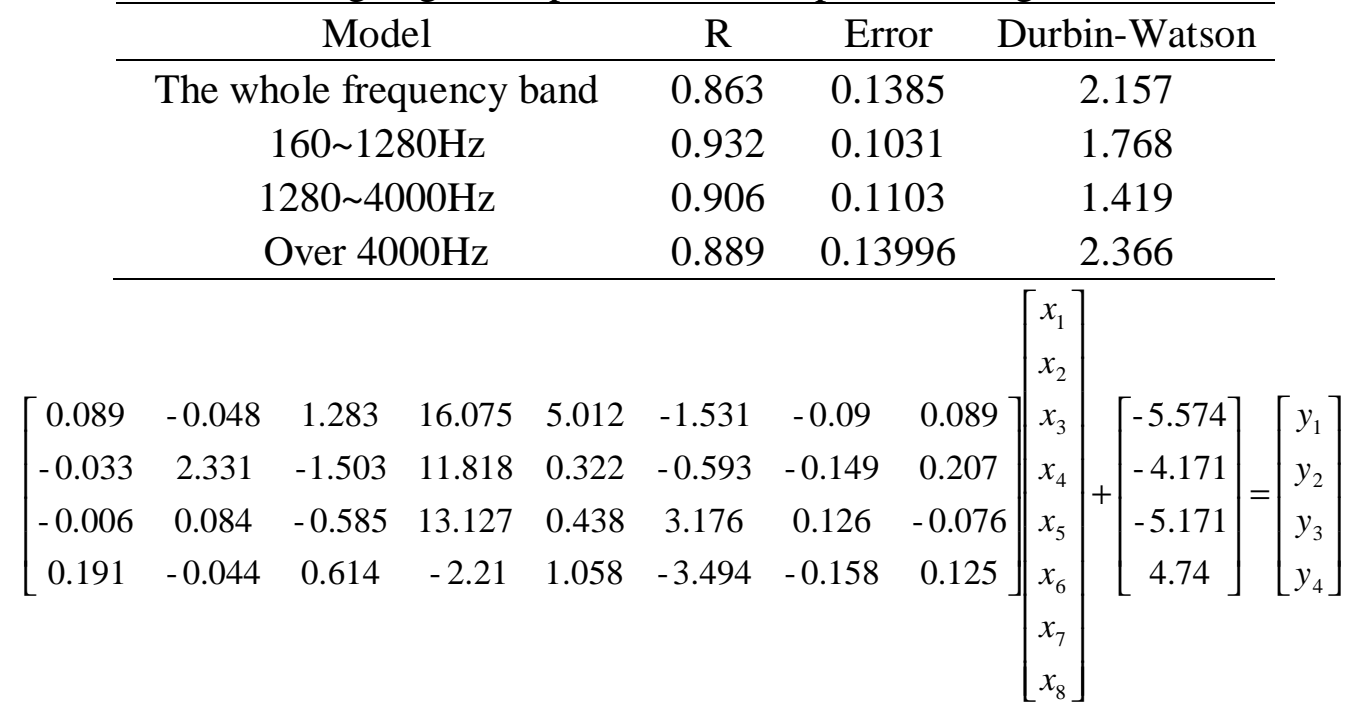

In the formula, $x_{1}$ is loudness; $x_{2}$ is sharpness; $x_{3}$ is harshness; $x_{4}$ is fluctuation; $x_{5}$ is tone; $x_{6}$ is $\mathrm{AI}$ index; $x_{7}$ is A-weighted sound pressure level; $x_{8}$ is linear sound pressure level; $y_{1}$ is subjective evaluation value in whole frequency band; $y_{2}$ is subjective evaluation value in $160-1280 \mathrm{~Hz} ; y_{3}$ is subjective evaluation value in $1280-4000 \mathrm{~Hz} ; y_{4}$ is subjective evaluation value in above $4000 \mathrm{~Hz}$.

After MLR model is established for the whole frequency band, $160-1280 \mathrm{~Hz}, 1280-4000 \mathrm{~Hz}$, and above $4000 \mathrm{~Hz}, 19-24$ noise samples are used to test accuracy of MLR model.

Objective parameter values of 19-24 noise samples are substituted into Formula (2) to gain predicted value of multivariate linear model. The predicted value is compared with subjective evaluation value, as shown in Table 3.

Table 3 Error Analysis

\begin{tabular}{ccccccc}
\hline Error & 19 & 20 & 21 & 22 & 23 & 24 \\
\hline$y_{1}[\%]$ & 37.15 & 6.27 & 24.79 & 31.05 & 13.12 & 39.22 \\
$y_{2}[\%]$ & 1.37 & 52.02 & 4.20 & 28.78 & 66.11 & 70.00 \\
$y_{3}[\%]$ & 2.22 & 26.51 & 39.03 & 21.80 & 32.91 & 80.00 \\
$y_{4}[\%]$ & 22.29 & 40.23 & 50.30 & 30.00 & 47.63 & 144.09 \\
\hline
\end{tabular}

It can be seen from Table 3 that, error values of the four models are large, and error stability is also not good. This is because linear relation between auditory sense of human ear and human subjective evaluation of sound quality is not strong, and non-linear relationship exists.

Sound quality evaluation model based on BP neural network. BP algorithm flow chart includes BP neural network construction, BP neural network training and BP neural network prediction $[9,10]$. The whole network contains two states: learning state and working state. Firstly, training data are applied to construct appropriate BP neural network. With given input value and output value, an appropriate neural network is predicted so that the error between network output value and actual output value is the smallest [11]. The test samples are inputted by such neural network to predict network testing effect.

The input of test samples 19-24 is substituted into well-trained BP neural network to enter verification stage of BP neural network. Then, predicted value of BP neural network is gained. The prediction results of samples 19-24 are shown in Table 4.

It is found from Table 4 that relative to MLR model, the error of BP neural network decreases a lot. But the error value is still large and there is certain distance with the predicted effect. 
Table 4 Predication results of BP neural network

\begin{tabular}{ccccccc}
\hline Frequency band & 19 & 20 & 21 & 22 & 23 & 24 \\
\hline Whole & 0.6472 & 0.8714 & 0.6349 & 0.1656 & 1.0016 & 0.1671 \\
$160-1280 \mathrm{~Hz}$ & 0.6225 & 0.5212 & 0.4021 & 0.1547 & 0.408 & 0.1388 \\
$1280-4000 \mathrm{~Hz}$ & 0.6353 & 1.0974 & 0.7177 & 0.2601 & 1.2587 & 0.1637 \\
Above $4000 \mathrm{~Hz}$ & 0.5398 & 0.9266 & 0.4011 & 0.352 & 0.6838 & 0.2921 \\
\hline
\end{tabular}

Sound quality evaluation model based on RBF neural network. Matlab is used to compile relevant RBF neural network program. RBF network took box equipped in Matlab is called. After input and output data are set well, RBF neural network is established through the order net=newrb. After the model is set up, the network will automatically carry out data training and set relevant neuron weight coefficient. Network training is achieved through the data of 1-18 noise samples. 19-24 samples are used to verify network prediction error [12].

The input of test samples 19-24 is substituted into well-trained RBF neural network to enter network verification stage. Then, predicted value of RBF neural network is gained. Predication results of samples 19-24 are shown in Table 5.

Table 5 Predication results of RBF model

\begin{tabular}{ccccccc}
\hline Frequency band & 19 & 20 & 21 & 22 & 23 & 24 \\
\hline Whole & 0.5734 & 0.8582 & 0.5669 & 0.2225 & 0.9816 & 0.0596 \\
$160-1280 \mathrm{~Hz}$ & 0.5212 & 0.9202 & 0.4595 & 0.1162 & 0.8321 & 0.0113 \\
$1280-4000 \mathrm{~Hz}$ & 0.7353 & 0.9097 & 0.7582 & 0.3423 & 0.8582 & 0.0596 \\
Above $4000 \mathrm{~Hz}$ & 0.6588 & 0.8253 & 0.7204 & 0.3802 & 1.0438 & 0.0506 \\
\hline
\end{tabular}

Contrastive analysis of prediction of three evaluation models. To objectively quantify the feeling of subjective preference evaluation and verify model accuracy, MLR model of objective evaluation, BP neural network model and radial basis function model are established. The test samples are substituted into the three models to gain error broken line graph of three models, as shown in Fig.2.
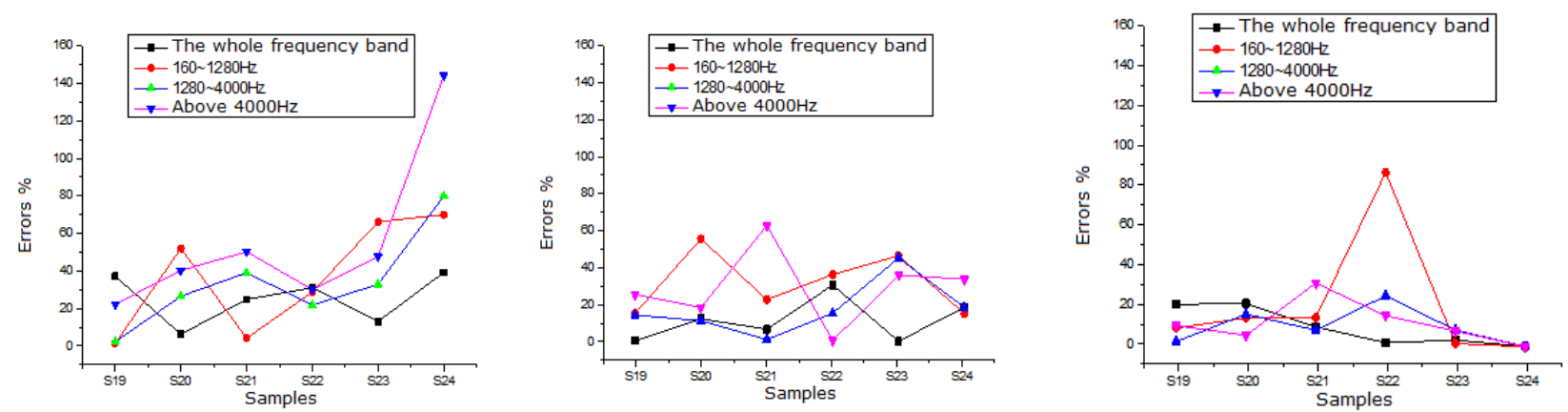

Fig. 2 Error broken line graph of three models

It is known from Fig.2 that, four broken lines of multivariate linear model farther deviate from $\mathrm{X}$ axis. This indicates the errors in the four frequency bands are large. In particular, the error of samples above $4000 \mathrm{~Hz}$ is large. In BP neural network, the distance between four broken lines and X axis reduces, relative to multivariate linear model. The prediction effect for the whole frequency band and $1280-4000 \mathrm{~Hz}$ frequency band is superior to $160-1280 \mathrm{~Hz}$ and above $4000 \mathrm{~Hz}$. In BF prediction model, many multivariate linear models and BP neural network are near $\mathrm{X}$ axis. This indicates the error further reduces. The error of sample 22 in $160-1280 \mathrm{~Hz}$ is large. On the whole, prediction effect of RBF model is superior to multivariate linear model and BP neural network model. 


\section{Summary}

This paper studies fundamentals and setup steps of MLR, BP neural network and RBF network and carries out contrastive analysis. Finally, the following conclusions are drawn.

For MLR model, the error above $4000 \mathrm{~Hz}$ is the largest, and the error of the whole frequency band is small. But the error values of four frequency bands are large, and error stability is not good. But this method is simple and has fast calculation speed, so it is suitable for linear problem analysis.

For BP neural network model, the error of the whole frequency band is the smallest, and the error in $160-1280 \mathrm{~Hz}$ is large. But on the whole, it further approaches $\mathrm{X}$ axis. This shows relative to MLR model, the error reduces greatly. However, the error is still large and there is certain distance with the ideal prediction effect. This model is suitable for solving large sample problem with complex internal mechanism.

The error of RBF neural network model in the whole frequency band and the other three frequency bands reduces a lot, compared with MLR model and BP neural network model. Except sample 22 , error range of other samples is within $20 \%$. The error value is stationary. Thus, RBF neural network model can well predict sound quality under such experimental conditions. The model does not need iteration, has fast learning speed, high prediction accuracy and small relative error, so it is suitable for nonlinear problem analysis of small samples.

\section{Acknowledgements}

This work was financially supported by both National Natural Science Foundation of China (51575238) and the Jiangsu Colleges and Universities Natural Science Research Project (15KJB580001).

\section{References}

[1] Xiaoping Chen: Acoustics and Psychoacoustics (Posts \& Telecom Press, China 2010), in Chinese

[2] Blauert J: submitted to Proc. Inter Noise'86 (1986)

[3] Yang Pan and Zihou Meng: submitted to ACTA ACUSTICA (2013), in Chinese

[4] Huiyuan Liu, Jieping Gu and Zihou Meng: submitted to Technical Acoustics (2008), in Chinese

[5] Jie Zhang and Dongxing Mao: submitted to Science China (2010), in Chinese

[6] D.X. Mao, W.Z Yu, Z.M Wang: submitted to ACTA ACUSTICA (2005)

[7] MAO Dong-xing, WANG Yong: submitted to Technical Acoustics (2006)

[8] Korol, Renee M, Canham, Peter B, Lucas, Alexandra R: submitted to Multiple regression analysis (2005)

[9] Sadeghi, B.H.M.: submitted to Journal of Materials Processing Technology (2000)

[10] Jianbing Yang: submitted to Wireless Internet Technology (2014), in Chinese

[11] Xiumin Shen, Shuguang Zuo, Lvchang He, Shiwei Zhang, Lin Li: submitted to Technical Acoustics (2009)

[12] Lili Su, Research on Vehicle Interior Sound Quality subjective and Objective Evaluation and Active Control Method. Jilin University, Jilin, 2012, in Chinese 